\title{
Transarterial Onyx Embolization of Cranial Dural Arteriovenous Fistulas: Long-Term Follow-Up
}

\author{
R.V. Chandra, T.M. Leslie-Mazwi, B.P. Mehta, A.J. Yoo, J.D. Rabinov, J.C. Pryor, J.A. Hirsch, and R.G. Nogueira
}

\begin{abstract}
BACKGROUND AND PURPOSE: Endovascular therapy with liquid embolic agents is a common treatment strategy for cranial dural arteriovenous fistulas. This study evaluated the long-term effectiveness of transarterial Onyx as the single embolic agent for curative embolization of noncavernous cranial dural arteriovenous fistulas.
\end{abstract}

MATERIALS AND METHODS: We performed a retrospective review of 40 consecutive patients with 41 cranial dural arteriovenous fistulas treated between March 2006 and June 2012 by using transarterial Onyx embolization with intent to cure. The mean age was 57 years; one-third presented with intracranial hemorrhage. Most (85\%) had cortical venous drainage. Once angiographic cure was achieved, long-term treatment effectiveness was assessed with DSA and clinical follow-up.

RESULTS: Forty-nine embolization sessions were performed; $85 \%$ of cranial dural arteriovenous fistulas were treated in a single session. The immediate angiographic cure rate was $95 \%$. The permanent neurologic complication rate was $2 \%$ (mild facial palsy). Thirty-five of the 38 patients with initial cure underwent short-term follow-up DSA (median, 4 months). The short-term recurrence rate was only $6 \%$ (2/35). All patients with occlusion at short-term DSA undergoing long-term DSA (median, 28 months) had durable occlusion. No patient with long-term clinical follow-up (total, 117 patient-years; median, 45 months) experienced hemorrhage.

CONCLUSIONS: Transarterial embolization with Onyx as the single embolic agent results in durable long-term cure of noncavernous cranial dural arteriovenous fistulas. Recurrence rates are low on short-term follow-up, and all patients with angiographic occlusion on short-term DSA follow-up have experienced a durable long-term cure. Thus, angiographic cure should be defined at short-term follow-up angiography instead of at the end of the final embolization session. Finally, long-term DSA follow-up may not be necessary if occlusion is demonstrated on short-term angiographic follow-up.

ABBREVIATIONS: $C D A V F=$ cranial dural arteriovenous fistula; $C V D=$ cortical venous drainage

$\mathbf{E}^{\mathrm{n}}$ ndovascular therapy is commonly used for the treatment of noncavernous cranial dural arteriovenous fistulas (cDAVFs). Cyanoacrylates, ethyl alcohol, coils, and particles can be used alone or in combination via transarterial, transvenous, or occasionally direct percutaneous treatment routes. There is no US

Received September 17, 2013; accepted after revision December 11.

From the Neuroradiology and Neurovascular Surgery Services, Departments of Diagnostic Imaging and Surgery (R.V.C.), Monash University, Melbourne, Victoria, Australia; Neuroendovascular Service (T.M.L.-M., B.P.M., A.J.Y., J.D.R., J.A.H.), Department of Neurology (T.M.L.-M., B.P.M.), and Department of Radiology (A.J.Y., J.D.R., J.A.H.), Division of Diagnostic Neuroradiology, Massachusetts General Hospital, Harvard Medical School, Boston, Massachusetts; Department of Interventional Neuroradiology (J.C.P.), Boston University, Boston, Massachusetts; and Neuroendovascular and Neurocritical Care Services and Neurology, Neurosurgery, and Radiology (R.G.N.), Marcus Stroke and Neuroscience Center, Grady Memorial Hospital, Emory University School of Medicine, Atlanta, Georgia.

Please address correspondence to Raul Gomes Nogueira, MD, 80 Jesse Hill Dr SE Room 398, Atlanta, GA 30303; e-mail: raul.g.nogueira@emory.edu

http://dx.doi.org/10.3174/ajnr.A3938
Food and Drug Administration-approved liquid embolic agent for the treatment of cDAVFs. The ethylene-vinyl alcohol copolymer liquid embolic system (Onyx; Covidien, Irvine, California) is FDA-approved for the presurgical embolization of brain arteriovenous malformations. Since Onyx has become available, transarterial embolization of cDAVFs by using Onyx as the sole endovascular embolic agent has become our preferred treatment strategy. This endovascular treatment approach represents an "off-label" use of the Onyx liquid embolic system.

The immediate occlusion rate in large cohort studies of patients treated with transarterial Onyx embolization ranges from $62 \%$ to $92 \%$, and short-term durable occlusion has been demonstrated. ${ }^{1-4}$ We have previously reported our short-term experience using Onyx in these patients ${ }^{5}$ and have compared the success of this technique with embolization using $n$-butyl cyanoacrylate. ${ }^{6}$ However, there currently remain no published data on the longterm effectiveness for embolization of cDAVFs by using Onyx, to 
Table 1: Baseline characteristics of study cohort ( $n=\mathbf{4 0}$ patients with 41 cDAVFs)

\begin{tabular}{lc}
\hline \multicolumn{1}{c}{ Characteristic } & No. of Patients \\
\hline Age (mean; range) & $57(30-79)$ \\
Male (\%) & $21(53 \%)$ \\
Presentation & \\
Tinnitus & $14(35 \%)$ \\
Hemorrhage & $13(33 \%)$ \\
Incidental/nonspecific headache & $10(25 \%)$ \\
$\quad$ syndrome & \\
Seizure & $2(5 \%)$ \\
Superficial siderosis & $1(2 \%)$ \\
Cognard grade & \\
I & $5(12 \%)$ \\
IIA & $3(7 \%)$ \\
IIB & $3(7 \%)$ \\
IIA $+B$ & $4(10 \%)$ \\
III & $15(37 \%)$ \\
IV & $11(27 \%)$ \\
Borden grade & \\
I & $8(15 \%)$ \\
II & $7(22 \%)$ \\
III & $26(63 \%)$ \\
\hline
\end{tabular}

our knowledge. Here we report our long-term angiographic occlusion rate and clinical follow-up in a cohort of noncavernous cranial DAVFs that were treated by using transarterial Onyx embolization with the intention of complete cure.

\section{MATERIALS AND METHODS \\ Patient Cohort}

Between March 2006 and June 2012, forty consecutive patients with 41 noncavernous cDAVFs treated by using transarterial Onyx embolization as the single embolic agent with the intention of complete cure were retrospectively identified. Baseline characteristics of the study cohort are described in Table 1 . This retrospective analysis was approved by the institutional review board of Massachusetts General Hospital.

\section{Embolization Procedure}

All procedures were performed with the patient under general anesthesia by using biplane angiographic units (Neurostar, Axiom Artis, and Artis zee; Siemens, Erlangen, Germany). Informed consent included the off-label use of Onyx as the primary embolization agent. All procedures were performed via the transfemoral approach by using coaxial techniques with continuous flushing of the catheters with heparinized saline (4000 $\mathrm{U}$ of heparin per liter). After initial diagnostic angiography, standard guide catheters were placed into the external carotid artery and dimethylsulfoxide-compatible microcatheters were directed into the target arterial pedicle. In most cases, a Marathon microcatheter (Covidien) was placed through a 5F MPC Envoy guide catheter (Codman \& Shurtleff, Raynham, Massachusetts) and navigated over an X-Pedion-10 (Covidien) or Mirage 0.008 microwire (Covidien) to reach the distal aspect of the target arterial pedicle. After microcatheter angiography confirmed an optimal position, the microcatheter was flushed with $5 \mathrm{~mL}$ of normal saline. The dead space of the microcatheter was subsequently filled with dimethylsulfoxide, and Onyx was injected during 90 seconds to fill the microcatheter and replace the dimethyl-sulfoxide in the dead
Table 2: Procedural characteristics of study cohort $(n=41$ DAVFs)

\begin{tabular}{lc}
\multicolumn{1}{c}{ Characteristic } & No. \\
\hline $\begin{array}{l}\text { No. of feeding pedicles (mean, range) } \\
\text { Total No. of embolization procedures }\end{array}$ & $4(1-10)$ \\
$\begin{array}{l}\text { No. of embolization procedures per patient } \\
\quad \text { (mean; range) }\end{array}$ & 49 \\
No. of single-stage embolizations & $1.2(1-3)$ \\
Complications ( $n=49$ embolization procedures) & $35(85 \%)$ \\
$\quad$ Technical events & $5(10 \%)$ \\
All clinical events & $7(14 \%)$ \\
Transient neurologic & $1(2 \%)$ \\
Permanent neurologic & $1(2 \%)$ \\
Death & 0 \\
\hline
\end{tabular}

space. Under continuous fluoroscopic guidance, Onyx was slowly infused into the target arterial pedicle by using a "thumb-tapping" technique to form an initial "plug" at the microcatheter tip. Excessive reflux was avoided to minimize challenges related to subsequent microcatheter removal and the risk of nontarget embolization. Once sufficient antegrade flow of Onyx was established, prolonged injections were performed. Intermittent injection pauses of between 30 and 120 seconds were used to avoid Onyx migration into nontarget areas and to facilitate redirection of newly injected Onyx to desired areas. In general, Onyx-34 was used for plug formation with subsequent injection of Onyx-18. Intermittent angiography through the guide catheter was occasionally performed during embolization to assess the extent of residual flow or areas of nontarget embolization. Angiographic cure was defined as formation of an attenuated Onyx cast in the target fistula and the proximal draining venous structures with no residual arteriovenous shunting. In 2 cases, Gelfoam (Phadia, Uppsala, Sweden) was injected into the superficial temporal and occipital arteries to further promote thrombosis of the scalp arteries after Onyx embolization.

\section{Follow-Up}

All patients were scheduled for short-term (3-month) follow-up angiography to confirm persistent occlusion of the cDAVF following the endovascular treatment course. If complete occlusion of the shunt was not achieved, further treatment (endovascular or surgical) was planned. No patients underwent radiosurgical treatment. Long-term (>12-month) angiographic follow-up was left to the discretion of the neurointerventionalist; early in the study period, 3-year angiography was scheduled.

\section{RESULTS}

\section{Patient Cohort}

Mean age was 57 years (range, 30-79 years), and one-third of patients presented with intracranial hemorrhage. Thirty-five (85\%) had cortical venous drainage (CVD); the classifications of Cognard et $\mathrm{al}^{7}$ and Borden et $\mathrm{al}^{8}$ are reported in Table 1.

\section{Embolization Safety Results}

Forty-nine embolization sessions were performed for 41 cDAVFs in 40 patients. The procedural characteristics are described in Table 2. Although the cDAVFs had multiple arterial feeding pedicles (mean, 4; range, 1-10), the mean number of embolization ses- 
Table 3: Procedural effectiveness at time of treatment, shortand long-term follow-up

\begin{tabular}{lc}
\hline \multicolumn{1}{c}{$\begin{array}{c}\text { Procedural Effectiveness } \\
\text { (No. of patients) }\end{array}$} & $\begin{array}{c}\text { Angiographic } \\
\text { Cure (No.) }\end{array}$ \\
\hline At end of final treatment (40) & $35(95 \%)$ \\
Short-term angiographic follow-up ${ }^{\text {a }}(35)$ & $33(94 \%)$ \\
Long-term angiographic follow-up $^{\text {b }(24)}$ & $24(100 \%)$ \\
\hline a Short-term follow-up occurred at a median of 4.2 months (2-12 months). \\
b Long-term angiographic follow-up occurred at a median of 28.2 months (12-63 \\
months).
\end{tabular}

sions per patient was 1.2 (range, $1-3)$. Thirty-five (85\%) cDAVFs were treated to cure in a single embolization session.

Technical procedural complications without clinical consequence occurred in 5 (10\%) of 49 embolization sessions-1 vertebral artery dissection, 1 occipital artery perforation during Onyx embolization that sealed, 1 microcatheter rupture in the middle meningeal artery, 1 instance of nontarget nonocclusive embolization into a superior sagittal sinus leaflet, and a single episode of intraprocedural thrombosis in the external carotid circulation during a treatment session despite heparinization, which raised the suspicion of heparin-induced thrombocytopenia. This particular procedure was aborted, and the cDAVF was cured on the third embolization session.

Adverse clinical events occurred in 7 (14\%) of 49 embolization sessions: focal alopecia from radiation injury, transient orbital chemosis of unclear etiology that resolved within 24 hours, 2 cranial nerve palsies (partial facial and maxillary nerve palsies) with near-complete and complete resolution in these 2 patients, transient intraprocedural bradycardia progressing to brief self-resolving asystole without clinical consequence during embolization through the artery of the falx cerebelli for a Cognard III cDAVF at the torcula, and an episode of immediate postextubation respiratory distress leading to secondary asystolic arrest. The patient was resuscitated and recovered without clinical consequences. The final event was pulmonary embolism occurring 4 days after embolization of a ruptured Cognard IV cDAVF in a 71-year-old man. The only permanent neurologic complication was in the patient with partial facial palsy. There were no procedure-related cerebral ischemic or hemorrhagic complications, nor procedural mortality.

\section{Embolization Effectiveness Results}

The effectiveness of the embolization procedure at the end of the final treatment, and at short- and long-term angiographic follow up is summarized in Table 3.

Immediate Results. At the end of the final session, angiographic cure was achieved in 38 of 40 (95\%) patients. Two patients were not cured after transarterial Onyx injection. A 72-year-old man with an incidental Cognard III cDAVF draining into a single cortical vein had minimal residual arteriovenous shunting at the end of 2 embolization sessions. His second embolization session was complicated by partial left maxillary and facial nerve palsies, and he underwent surgical closure of the shunt. His cranial nerve palsies had recovered by his 6-month clinical follow-up. The second patient, a 54-year-old woman, presented with pulsatile tinnitus from an extensive transverse-sigmoid sinus junction Cognard IIa cDAVF. Although significant reduction in shunting volume was achieved, her second embolization session was complicated by partial facial nerve palsy. Her pulsatile tinnitus became tolerable; thus, no further treatment was performed. Her facial nerve function recovered to almost normal, with only minimal residual palsy.

Short-Term Follow-Up. Thirty-five (92\%) of the 38 patients with immediate angiographic occlusion underwent short-term follow-up DSA at a median of 4.2 months (range, 2-12 months). Two patients $(6 \%)$ had recurrence of their cDAVF. One patient with recurrence of his Cognard IV cDAVF located at the torcula underwent an additional uncomplicated embolization session to angiographic cure, which was durable at repeat angiography at 10 months posttreatment. The second recurrence occurred in a patient with a Cognard IV cDAVF supplied by the right superficial temporal artery, which was treated with surgical occlusion.

Long-Term Follow-Up. Twenty-four (73\%) of the 33 patients with short-term angiographic occlusion underwent long-term (>12-month) DSA at a median of 28.2 months (range, 12-63 months). All patients (100\%) had durable occlusion of their cDAVF at 59 patient-years of follow-up. Long-term clinical ( $>12$-month) follow-up was available for 32 patients at a median of 44.8 months (12-80 months). No patient experienced intracranial hemorrhage during 117 patient-years of long-term follow-up.

\section{DISCUSSION}

Our results demonstrate the effectiveness of transarterial Onyx embolization as the single embolic agent for achieving long-term durable cure of noncavernous cDAVFs. In addition, in a cohort of patients in which one-third presented with intracranial hemorrhage and $85 \%$ demonstrated CVD, clinical outcomes were favorable, with minimal permanent complications and no patient experiencing intracranial hemorrhage during 117 patient-years of long-term follow-up.

Transarterial Onyx embolization has provided a new treatment paradigm for the care of patients with cDAVFs. The characteristics of the compound allow it to penetrate the multiple arterial branches that supply these lesions and occlude vascular passages into the proximal venous component, the ultimate treatment goal. Several large cohort studies by using transarterial Onyx as the primary embolization method have reported high initial cure rates and durable short-term angiographic occlusion. Immediate postembolization or short-term DSA cure rates range from $62 \%$ to $92 \% .^{1-4}$ Angiographic follow-up varied per series, with a median of 3-6 months. In 2 large cohort series, the short-term DSA occlusion rate was $100 \% .^{1,2}$ Therefore, these data provide satisfactory evidence that effective short-term occlusion with Onyx is readily achievable.

However, longer term angiographic and clinical follow-up data have not been published to validate the long-term effectiveness of this treatment strategy. In addition, there have been reports of recurrence of cured cDAVFs after transarterial treatment. ${ }^{9}$ Our data demonstrate that angiographic cure is only definitively confirmed after interval short-term angiography demonstrates cDAVF occlusion. In our cohort, 2 of 35 patients with immediate posttreatment angiographic occlusion had recurrence at short-term (median, 4.2 months) DSA. These recurrences 
could be potentially related to intraprocedural vasospasm, which could obscure residual areas of arteriovenous shunting, persistent microfistulas not visible immediately following the initial treatment, or insufficient penetration of Onyx into the proximal draining venous structures. However, once occlusion is demonstrated on shortterm DSA, all patients had durable angiographic occlusion at a median of 28.2 months (range, 12-63 months).

The ultimate clinical goal of treatment of cDAVFs is to alter the natural history, particularly with regard to the risk of intracranial hemorrhage. Large population studies reveal that approximately $30 \%$ of patients with CDAVF with CVD present with hemorrhage. ${ }^{10}$ The risks of early rebleeding can be high-in an earlier cohort of 20 patients with intracranial hemorrhage from cDAVFs, 7 patients (35\%) had radiologically confirmed rebleeding within the first 2 weeks after presentation. ${ }^{11}$ Long-term morbidity and mortality rates are elevated in patients with persistent CVD. In a cohort of 20 patients with persistent long-term CVD followed for a mean of 4.3 years, 7 patients (35\%) bled and 9 patients $(45 \%)$ died. ${ }^{12}$ Five of the 20 patients initially presented with intracranial hemorrhage -3 of these 5 patients rebled; 5 of 15 remaining patients developed hemorrhage. The hemorrhages during follow-up occurred at a median of 2.3 years (range, $0.3-5.4$ ) after the initial presentation. Despite treatment, cDAVFs with CVD are associated with excessive long-term mortality, ${ }^{13}$ making long-term clinical follow-up prudent. In our treated cohort, one-third presented with intracranial hemorrhage and 9 of these 13 patients had long-term (>12-month) clinical follow-up (median, 44.5 months; range, $12.3-81.3$ months; total, 34 patient-years). All are alive, and none experienced rebleeding.

It has been reported that patients presenting with benign (incidental/pulsatile tinnitus/orbital phenomena) symptoms from cDAVFs with CVD may be at lower annual risk for intracranial hemorrhage (approximately 1.5\%) compared with those presenting with aggressive neurologic symptoms. ${ }^{14-16}$ However, these estimates are based on small patient numbers or short follow-up periods. In the series by Strom et al, ${ }^{15} 1$ of 17 patients presenting with benign symptoms from persistent CVD bled 13 years later. In another series by Söderman et al, ${ }^{14}$ a complicated "time-at-risk" period was calculated by combining the time between symptom onset/nonangiographic diagnosis, the time between diagnostic angiography and treatment, and clinical follow-up. In the cohort of 53 patients who did not present with intracranial hemorrhage, 1 patient bled during a time-at-risk period of 67.1 patient-years, conferring an annual 1.5\% risk for intracranial hemorrhage. Despite the complex methodology and limitations, ${ }^{17}$ the per-patient follow-up period of 1.3 years is relatively short. In the most recent publication evaluating this patient cohort, 1 in 24 cDAVFs with benign presentation bled during a total of 23 patient-years of untreated follow-up, leading to an annual hemorrhage rate of $4.3 \% .{ }^{18}$ Of note, the range of follow-up for the entire cohort of 70 patients was 1 day to 13 years.

Most (24 of 40) of the patients in our cohort had benign presentation - pulsatile tinnitus, nonspecific headache syndrome, or incidental. Eighteen of 24 patients with benign presentation had CVD. In this cohort, the long-term risks of intracranial hemorrhage need to be balanced against the treatment risks. In the entire cohort, only 1 permanent neurologic complication (partial facial palsy) occurred in 40 patients who underwent 49 embolization sessions. This is within the $(0 \%-7.5 \%)$ permanent complication rate in the larger published cohorts treated with transarterial Onyx. ${ }^{1,3,4,19}$ While the natural history of patients with benign symptoms from cDAVFs with CVD remains unclear, we think that the high success rate coupled with a low morbidity rate from transarterial Onyx embolization mandates treatment to angiographic cure once CVD is discovered, for long-term hemorrhage protection.

Our study adds unique and important long-term effectiveness data supporting the use of transarterial Onyx for the embolization of cDAVFs. The strengths of our study include the long-term angiographic and clinical follow-up, the use of DSA to confirm durable occlusion, and the homogeneous and relatively large cohort of noncavernous cDAVFs treated with transarterial Onyx. The limitations are the retrospective study design and the 9 patients with cure on short-term follow-up DSA who did not undergo long-term DSA. This limitation reflected a change in our practice as we gathered experience during the study period. The presence of long-term durable angiographic occlusion in all patients with occlusion on short-term DSA follow-up during the study period led some of our neurointerventionists to perform MR angiography after short-term DSA follow-up.

\section{CONCLUSIONS}

Transarterial embolization with Onyx as the sole embolic agent results in durable long-term cure of noncavernous cranial DAVFs. Recurrence rates are low on short-term follow-up, and all patients with angiographic occlusion on short-term DSA follow-up have durable long-term cure. Thus, angiographic cure should be defined at short-term follow-up angiography instead of at the end of the final embolization session. Finally, long-term DSA follow-up may not be necessary if occlusion is demonstrated on short-term angiographic follow-up.

Disclosures: Albert J. Yoo—UNRELATED: Grants/Grants Pending: Penumbra, ${ }^{*}$ Comments: Core Imaging Lab for stroke trials. Johnny C. Pryor-RELATED: Support for Travel to Meetings for the Study or Other Purposes: Travel was paid when Dr Pryor arrived before the meeting to conduct animal research, consult, or teach colloquia prior to meeting; no travel occurred within 36 months of submission, Other: Massachusetts General Hospital was compensated for food and expenses for Onyx Colloquia; Dr Pryor had travel expenses paid directly for research and consultation at ev3 and University of California, Riverside in California; no such activities have occurred within 36 months of submission, UNRELATED: Stock/Stock Options: purchased stock in Chestnut Medical Technologies, which was purchased by ev3. Joshua A. Hirsch—UNRELATED: Consultancy: CareFusion, Aetrium, Comments: Both $>12$ months but $<36$ months ago, related to spine, Royalties: CareFusion, Comments: As above, Stock/Stock Options: Intratech, Comments: development-stage stroke company. Raul G. Nogueira—UNRELATED: Consultancy: Stryker Neurovascular (Principal Investigator of Trevo Versus Merci Retrievers for Thrombectomy Revascularisation of Large Vessel Occlusions in Acute Ischaemic Stroke 2 and DWI/PWI and CTP Assessment in the Triage of Wake-Up and Late Presenting Stroke Undergoing Neurointervention Trials), Covidien (Steering Committee for the SWIFT [Solitaire with the Intention for Thrombectomy] and SWIFT Prime Trials, Core Lab for the Solitaire FR Thrombectomy for Acute Revascularization Trial), Penumbra (Executive Committee for the Penumbra 3-D Trial); Rapid Medical (Data and Safety Monitoring Board). *Money paid to the institution.

\section{REFERENCES}

1. Abud TG, Nguyen A, Saint-Maurice JP, et al. The use of Onyx in different types of intracranial dural arteriovenous fistula. AJNR Am J Neuroradiol 2011;32:2185-91

2. Cognard C, Januel AC, Silva NA Jr, et al. Endovascular treatment of 
intracranial dural arteriovenous fistulas with cortical venous drainage: new management using Onyx. AJNR Am J Neuroradiol 2008;29:235-41

3. Hu YC, Newman CB, Dashti SR, et al. Cranial dural arteriovenous fistula: transarterial Onyx embolization experience and technical nuances. J Neurointerv Surg 2011;3:5-13

4. Lv X, Jiang C, Zhang J, et al. Complications related to percutaneous transarterial embolization of intracranial dural arteriovenous fistulas in 40 patients. AJNR Am J Neuroradiol 2009;30:462-68

5. Nogueira RG, Dabus G, Rabinov JD, et al. Preliminary experience with Onyx embolization for the treatment of intracranial dural arteriovenous fistulas. AJNR Am J Neuroradiol 2008;29:91-97

6. Rabinov JD, Yoo AJ, Ogilvy CS, et al. Onyx versus n-BCA for embolization of cranial dural arteriovenous fistulas. J Neurointerv Surg 2013;5:306-10

7. Cognard C, Gobin YP, Pierot L, et al. Cerebral dural arteriovenous fistulas: clinical and angiographic correlation with a revised classification of venous drainage. Radiology 1995;194:671-80

8. Borden JA, Wu JK, Shucart WA. A proposed classification for spinal and cranial dural arteriovenous fistulous malformations and implications for treatment. J Neurosurg 1995;82:166-79

9. Adamczyk P, Amar AP, Mack WJ, et al. Recurrence of "cured" dural arteriovenous fistulas after Onyx embolization. Neurosurg Focus 2012;32:E12

10. Piippo A, Niemela M, van Popta J, et al. Characteristics and longterm outcome of 251 patients with dural arteriovenous fistulas in a defined population. J Neurosurg 2013;118:923-34

11. Duffau H, Lopes M, Janosevic V, et al. Early rebleeding from intra- cranial dural arteriovenous fistulas: report of 20 cases and review of the literature. J Neurosurg 1999;90:78-84

12. van Dijk JM, terBrugge KG, Willinsky RA, et al. Clinical course of cranial dural arteriovenous fistulas with long-term persistent cortical venous reflux. Stroke 2002;33:1233-36

13. Piippo A, Laakso A, Seppa K, et al. Early and long-term excess mortality in 227 patients with intracranial dural arteriovenous fistulas. J Neurosurg 2013;119:164-71

14. Söderman M, Pavic L, Edner G, et al. Natural history of dural arteriovenous shunts. Stroke 2008;39:1735-39

15. Strom RG, Botros JA, Refai D, et al. Cranial dural arteriovenous fistulae: asymptomatic cortical venous drainage portends less aggressive clinical course. Neurosurgery 2009;64:241-47, discussion 247-48

16. Zipfel GJ, Shah MN, Refai D, et al. Cranial dural arteriovenous fistulas: modification of angiographic classification scales based on new natural history data. Neurosurg Focus 2009;26:E14

17. van Dijk JM, Terbrugge KG, Willinsky RA, et al. The natural history of dural arteriovenous shunts: the Toronto experience. Stroke 2009; 40:e412, author reply e413-14

18. Bulters DO, Mathad N, Culliford D, et al. The natural history of cranial dural arteriovenous fistulae with cortical venous reflux: the significance of venous ectasia. Neurosurgery 2012;70:312-18, discussion 318-19

19. Stiefel MF, Albuquerque FC, Park MS, et al. Endovascular treatment of intracranial dural arteriovenous fistulae using Onyx: a case series. Neurosurgery 2009;65(6 suppl):132-39, discussion 139-40 